\title{
EXPLORATIONS ON THE EARTH'S RELATIVISTIC GRAVITY FIELD
}

\author{
Wenbin Shen \\ Section of Physical Geodesy, Graz Technical University, A-8010 Graz, Austria
}

\section{MEASURE STANDARDS}

According to Newtonian theory, space and time are absolute, and consequently, once a length measure standard and a time measure standard are chosen, they keep invariant. According to relativity however, the length and time measure standards may vary with different positions and different reference systems. This implies that the observed values at points $\mathrm{A}$ and $\mathrm{B}$ are not absolute. Hence, we must establish a "common measure standard system", into which all observed values must be transformed.

Time measure is realized by the time keeping of a precise clock. In the viewpoint of relativity, the running rate of a clock depends not only on its velocity (the state of motion) but also its position at which there exists a gravitational potential. Hence, generally, clocks located at two different points on (above or under) the earth's surface run with different rates. However, we can assume that there exists an ideal clock at infinity at rest with respect to the cosmic microwave background (cf. Shen 1997). This clock is not influenced by the earth's rotation, neither influenced by gravitation nor gravitational potential. Under this assumption, we can establish the following relation:

$$
T=T_{0}\left(1-\frac{G M}{r}-\frac{1}{2} v^{2}\right),
$$

where $T_{0}$ and $T$ are the time keepings of ideal clock and gronud clock respectively, $M$ is the earth's mass, $G$ is the gravitational constant, $r$ is the distance from the geocenter to the ground clock, and $v$ is the velocity of the gronud clock with respect to the geocentric star-fixed system. Equation (1) shows that the ground clock runs slower than the ideal clock does.

Because of the existence of the gravitational field, the length of a measure rod depends on the directions along which the rod is set. Suppose the gravitational field consists of two parts: the main part that is caused by a uniform sphere (with the earth's mass), whose metric is the Schwarzschild metric $g_{\mu \nu}$ (cf. Weinberg 1972); and the perturbation part that is caused by irregular masses, whose metric is supposed to be $h_{\mu \nu}$. According to the Schwarzschild metric, the rod will not be influenced by gravitational field when it is set along the transverse direction of a spherical field, denoted as $L_{t}$, and it will be influenced by gravitational field when it is set along the radial direction of a spherical field, denoted as $L_{r}$ (cf. Lorentz et al. 1923). Suppose there is at infinity a rod $L_{0}$ at rest, 
which is neither influenced by gravitation nor the earth's motion, and consequently it can be considered as an ideal length. Then we have (cf. Lorentz et al. 1923):

$$
L_{t}=L_{0}, \quad L_{r}=\left(1-\frac{G M}{r}\right) L_{0}
$$

The earth's rotation is not taken into account in the Schwarzschild metric. In fact, if we resort to the Kerr metric (cf. Weinberg 1972), we can still get the above result (cf. Shen 1997).

\section{GRAVITATION}

In Newtonian theory, gravitation is related neither to the state of the particle's motion nor to the state of the gravitational source's motion. In general relativity however, gravitational effect is related to both the states of the particle's motion and the gravitational source's motion. However, the nature of gravitation is still a great mystery. We do not know why the gravitational field appears as it does. But one fact is definite: gravitational fields originate from gravitational masses, just as electromagnetic fields originate from (electric) charges. Furthermore, it is found that gravitational fields can be expressed geometrically. Gravitation causes the curvature of spacetime; and the curvature of spacetime characterizes some aspects of the gravitational field. Note that we cannot say that the spacetime curvature is gravitation. Essentially, gravitation is one kind of physical phenomenon arising from the mass sources.

\section{INERTIA}

When a body has an acceleration with respect to an inertial reference system, it "feels" a force which is opposite to the direction of the acceleration. Based on the well-known rotation experiment of the water bucket, Newton (1686) stated that inertia arises from the acceleration of the body relative to the absolute space. However, Mach (1883) stated that inertia arises from the acceleration relative to the total matter of the universe (cf. Mach 1883). Who is right?

Trying to answer this question, let us consider the following Gedankenexperiment (cf. Shen 1996): we assume that the universe is completely empty. If Mach is right, there will not exist any inertial force at all. However; if Newton is right, inertial forces will exist. In fact, once an acceleration reference system is introduced, an inertial force followes; once a rotating reference system is introduced, the inertial centrifugal force, Coriolis force and Euler force are introduced. It seems that inertial forces are not related to the total matter of the universe. Unfortunately, since there does not exist the empty universe that we have assumed, we cannot make a final judgement: Who is correct, Newton or Mach?

\section{GRAVITY}

Generally, we may define gravity as follows (cf. Shen 1996):

Referring to a celestial body, at an arbitrary point $\mathbf{P}$ (no matter outside or inside the body), gravity is the superposition of the gravitation and the inertial forces caused by the celestial body, provided that the point $\mathbf{P}$ is at the moment rigidly fixed to the body. 
Any inertial force is due to the use of a non-inertial reference system. In the geocentric earth-fixed system (GES), which is a non-inertial system, there exists a centrifugal force field. However, in the geocentric star-fixed system (GSS), which is a quasi-inertial system, there does not exist centrifugal force field. The reference system does neither influence gravitational fields, nor the curvature of spacetime. Reference system effects can be calculated theoretically, provided that a definite reference system is chosen. Hence, once the earth's gravitational field is determined, the gravity field is determined.

In practice, (generally) the observed quantities contain the reference system effects. Consequently, it is necessary to discuss the coordinate transformation between reference systems. Once a reference system is transformed, the corresponding metric $g_{\mu \nu}$ as well as the Christofel symbols $\Gamma_{\mu \nu}^{\lambda}$ will change. Since $g_{\mu \nu}$ and $\Gamma_{\mu \nu}^{\lambda}$ have the characters of gravitational potential and gravitation respectively (cf. Weinberg 1972), and are related to the choice of the reference systems, they can be considered as having the characters of the geopotential and gravity respectively.

Suppose there exists a gravitational field caused by a non-rotating uniform sphere. Then, the field is expressed by the Schwarzschild metric. In a spberical coordinate system $\mathrm{S}$ (suppose $\mathrm{S}$ is the geocentric star-fixed system GSS), the spacetime interval can be expressed as

$$
d \tau^{2}=\left(1-\frac{2 M}{r}\right) d t^{2}-\left(1-\frac{2 M}{r}\right)^{-1} d r^{2}-r^{2} d \theta^{2}-r^{2} \sin ^{2} \theta \varphi^{2}
$$

Now we consider the metric form expressed in another coordinate system $S^{\prime}$, which is chosen in such a way that it rotates with a constant angular velocity $\vec{\omega}$ with respect to $S$ (suppose $S^{\prime}$ is the geocentric earth-fixed system GES), and its origin and the third space axis always coincide with the origin and the third space axis of $\mathrm{S}$ respectively. Suppose point $\mathrm{P}$ has coordinates $(t, r, \theta, \varphi)$ in system $\mathrm{S}$ and coordinates $\left(t^{\prime}, r^{\prime}, \theta^{\prime}, \varphi^{\prime}\right)$ in system $\mathrm{S}^{\prime}$. Obviously, $r^{\prime}$ and $\theta^{\prime}$ are equal to $r$ and $\theta$ respectively, i.e.,

$$
r^{\prime}=r, \quad \theta^{\prime}=\theta
$$

and $\varphi^{\prime}$ varies with time, given by the following equation:

$$
\varphi^{\prime}=\varphi-\omega t
$$

However, we will encounter a difficulty in considering the transformation between the time coordinates $t$ and $t^{\prime}$ (cf. Shen 1996). Not going into details we just point out that it is reasonable to choose the following transformation equation (cf. M $\phi$ ller 1972; Shen 1996):

$$
t^{\prime}=t
$$

Then, we can write the coordinate transformation between $\mathrm{S}$ and $\mathrm{S}^{\prime}$ as follows:

$$
t^{\prime}=t, r^{\prime}=r, \theta^{\prime}=\theta, \varphi^{\prime}=\varphi-\omega t .
$$

Substituting (4) into (3) we get

$$
\begin{aligned}
d \tau^{\prime 2} \equiv & d \tau^{2} \\
= & \left(1-\frac{2 M G}{r^{\prime}}\right) d t^{\prime 2}-\left(1-\frac{2 M G}{r^{\prime}}\right)^{-1} d r^{2}-r^{\prime 2} d \theta^{2}-r^{\prime 2} \sin ^{2} \theta^{\prime}\left(d \varphi^{\prime}+\omega d t^{\prime}\right)^{2} \\
= & \left(1-\frac{2 M G}{r^{\prime}}-r^{\prime 2} \omega^{2} \sin ^{2} \theta^{\prime}\right) d t^{2}-\left(1-\frac{2 M G}{r^{\prime}}\right)^{-1} d r^{\prime 2}-r^{\prime 2} d \theta^{\prime 2} \\
& -r^{\prime 2} \sin ^{2} \theta^{\prime} d \varphi^{\prime 2}-2 r^{\prime 2} \omega \sin ^{2} \theta^{\prime} d \phi^{\prime} d t^{\prime} .
\end{aligned}
$$


Observed in system S, $g_{\mu \nu}$ is given by (3), based on which the constructed Christofel symbols $\Gamma_{\mu \nu}^{\lambda}$ have only the character of gravitation. However observed in system $\mathrm{S}^{\prime}, g_{\mu \nu}$ is given by (5), based on which the constructed Christofel symbols $\Gamma_{\mu \nu}^{\lambda}$ contain not only gravitational effects but also the inertial effects. This can be seen clearly by comparing $g_{\mu \nu}$ terms contained in (3) and (5). If we further construct the Riemann tensor $R_{\mu \nu \alpha}{ }^{\beta}$, we will find that no matter which kind of reference system is chosen, the Riemann tensor $R_{\mu \nu \alpha}^{\beta}$ is not influenced (cf. Shen and Moritz 1996): all the effects stemming from the different choices of reference systems such as the centrifugal force and Coriolis force vanish. In other words, the choice of the reference system does not influence the nature of spacetime (gravitation). Hence, we conclude that gravitation has the essential character, and the gravity is an additional concept. Further discussions are found in Shen (1997).

\section{REFERENCES}

Lorentz H A, Einstein A, Minkowski H, Weyl H (1923), The Principle of Relativity: A Collection of Original Memories. Methuen, London.

Mach E (1883), Die Mechanik in Ihrer Entwicklung (6. Auflage 1908), F.A.Brockhaus, Leipzig; The Science of Mechanics (transl. by T.J.McCormack, 5th ed. 1942). Open Court, LaSalle, III.

M $\phi$ ller C (1972), The Theory of Relativity (2nd ed., 1st ed. publ. 1952). Oxford University Press, Oxford.

Newton I (1687), Philosophiae Naturalis Principia Mathematica (English transl. by Andrew Motte, revised and annotated by F.Cajori). University of California Press (1966), California.

Shen W (1996), On the Separability of Gravitation and Inertia According to General Relativity. Dissertation, Graz Technical University, Graz.

Shen W (1997), Relativistic Physical Geodesy. Habilitation. Institute of Theoretical Geodesy, Graz Technical University, Graz.

Shen W, Moritz H (1996), On the Separation of Gravitation and Inertia and the Determination of the Relativistic Gravity Field in the Case of Free Motion. The Journal of Geodesy, Vol. 70, pp. 633-644.

Weinberg S (1972), Gravitation and Cosmology. John Wiley \& Sons, New York. 